\title{
Abordagem terapêutica dos hemangiomas cutâneos na infância*
}

\author{
Therapeutic management of skin hemangiomas in children
}

\author{
Adriana Maria da Silva Serra ${ }^{1}$ \\ Acimar G. da Cunha Júnior ${ }^{3}$
}

\author{
Flávia Machado Gonçalves Soares ${ }^{2}$ \\ Izelda Maria Carvalho Costa ${ }^{4}$
}

Resumo: Fundamentos: Hemangioma é um tumor benigno de células endoteliais comum na infância e de involução espontânea. OBJETIVO: Avaliar os tratamentos utilizados em 122 pacientes com hemangiomas cutâneos, tratados no Hospital Universitário de Brasília, de março de 2000 a dezembro de 2006.

MÉTODOs: Utilizou-se coleta de dados em prontuários e aplicação de questionários aos pais. Foram analisados gênero, fatores pré-natais e perinatais, características clínicas, tipo de tratamento e resultados.

Resultados: A razão de sexo (F: M) foi de 1,5:1. 42, em pacientes que apresentavam hemangiomas superficiais, 13 profundos e 67 mistos; em 7 pacientes, havia associação com síndromes. Em 79 pacientes, localizavam-se no polo cefálico. Já em 98/122 dos pacientes, foram submetidos a tratamento único e 24/122 a múltiplos. No primeiro grupo, foi utilizada conduta expectante em 38 pacientes, compressão em 3, corticoide sistêmico em 18, corticoide intralesional em 13, corticoide tópico em 4, cirurgia convencional em 12, criocirurgia em 7, luz pulsada em 1 e imiquimod em 2. No segundo grupo, 15 fizeram 2 tipos de tratamentos e 9 necessitaram de 3 ou mais tratamentos. CONCLUSÃo: Os dados obtidos concordam com a literatura mundial, quanto a sexo e localização da lesão. A incidência de hemangiomas presentes ao nascimento foi maior que em outras publicações. Os resultados terapêuticos obtidos foram comparáveis aos publicados na literatura. A identificação dos hemangiomas que necessitam de tratamento, em que momento adequado para intervenção e a melhor opção terapêutica devem ser considerados.

Palavras-chave: Avaliação de resultado de intervenções terapêuticas; Cirurgia geral; Corticosteroides; Criocirurgia; Hemangioma; Terapêutica; Terapia combinada

Abstract: BACKGROUND: Hemangiomas are benign, self-involuting tumors of endothelial cells that are common in childhood.

OвJестіves: To evaluate the treatment of 122 patients with skin hemangiomas treated at a teaching hospital in Brasilia, Brazil between March 2000 and December 2006.

MATERIAL AND METHODS: Data were collected from the patients' medical records and questionnaires were applied to the children's parents. Variables analyzed were: gender, pre- and perinatal factors, clinical characteristics of the lesions, type of treatment and outcome.

RESUlts: A male-to-female ratio of 1.5:1 was found. In 42 patients, hemangiomas were superficial, while in 13 cases they were deep and in 67 patients lesions were mixed. In 7 patients, the lesions were associated with syndromes. In 79 patients, hemangiomas were in the cephalic region. Of the 122 patients, 98 were submitted to one single treatment, while 24 required multiple forms of treatment. In the first group, expectant management was the conduct in 38 patients, compression in 3 cases, systemic corticotherapy in 18 patients, intralesional corticotherapy in 13, topical steroids in 4 cases, conventional surgery in 12 patients, cryosurgery in 7 , pulsed light in one case and imiquimod in 2 patients. In the second group of 24 patients submitted to multiple treatment modalities, 15 required two types of treatment and 9 needed three or more.

CONCLUSIONS: The data obtained in this study were in agreement with results in the literature with respect to gender and the site of the lesions. The incidence of hemangiomas present at birth was higher than rates published in the literature. The therapeutical results obtained were comparable with data from other published studies. Identification of hemangiomas that require treatment, as well as the right moment for intervention and the best therapeutic option for each case, are factors that need to be taken into consideration.

Keywords: Corticosteroids; Combined therapy; Cryosurgery; Outcome of therapeutic interventions; General surgery; Hemangioma; Therapeutics

Recebido em 21.9.2009.

Aprovado pelo Conselho Consultivo e aceito para publicação em 21.09.09.

* Trabalho realizado no Ambulatório de Dermatologia Pediátrica e Ambulatório de Cirurgia Pediátrica do Hospital Universitário de Brasília (HUB) - Brasília (DF), Brasil. Conflito de interesse: Nenhum / Conflict of interest: None

Suporte financeiro: Nenhum / Financial funding: None

Mestre em Ciências da Saúde pela Universidade de Brasília. Médica da Secretaria de Saúde do Distrito Federal - Brasília (DF), Brasil.

Mestranda em Ciências da Saúde pela Universidade de Brasília. Médica da Secretaria de Saúde do Distrito Federal - Brasília (DF), Brasil.

Doutor em Ciências da Saúde pela Universidade de Brasília. Médico da Secretaria de Saúde do Distrito Federal - Brasília (DF), Brasil.

Professora Adjunta de Dermatologia da Universidade de Brasília (DF), Brasil. Coordenadora do Ambulatório de Dermatopediatria do HUB/UNB. 


\section{INTRODUÇÃO}

Hemangioma é um tumor benigno de células endoteliais comum na infância, com história natural de crescimento rápido, nos primeiros meses de vida, seguindo-se fase de regressão lenta até regressão parcial ou total da lesão.'

O termo hemangioma já foi empregado no passado, de maneira ampla e indiscriminada. Atualmente é usado para descrever um grupo específico de tumor vascular, que surge na infância, com características clínicas, histopatológicas, evolução e prognóstico próprios. Os hemangiomas devem ser diferenciados das malformações vasculares. Essas últimas estão presentes ao nascimento, já que têm um crescimento proporcional ao da criança e possuem células endoteliais achatadas na histologia. ${ }^{2}$ Já os tumores vasculares são neoplasias da vasculatura (proliferação vascular) e incluem os hemangiomas da infância. ${ }^{3}$ Em pequena minoria de pacientes, a associação entre tumores vasculares e malformações vasculares pode ocorrer. ${ }^{4}$

O diagnóstico dos hemangiomas é feito baseado na história clínica e no exame físico do paciente, em 95\% dos casos. ${ }^{1,3}$ A sua patogênese ainda não é bem conhecida, mas estudos sugerem uma desregulação da homeostase vascular, por erro no desenvolvimento, ocorrido no primeiro trimestre da gestação. ${ }^{4,5}$

De 30 a $50 \%$ dos recém-nascidos (RN) apresentam ao nascimento um sinal precursor. ${ }^{3} \mathrm{~A}$ fase de crescimento do hemangioma ocorre nas primeiras semanas de vida, com ápice entre o $3^{\circ}$ e $6^{\circ}$ mês de vida, podendo se estender até 18 a 24 meses. Posteriormente, entra em uma fase quiescente, persistindo por alguns meses. A fase de involução lenta se inicia entre o $12^{\circ}$ e $18^{\circ}$ mês de vida. A fase de regressão completa ocorre em uma taxa de regressão de $10 \%$ ao ano, sendo que, até os 5 anos, $50 \%$ dos hemangiomas regrediram. As hemangiomas não resolutas, até o $6^{\circ}$ ano de vida, terão alterações residuais. As alterações residuais mais comuns são: as telangiectasias, rugas atróficas, descoloração amarelada, pele redundante, cicatriz e alopecia.

Em recém-nascidos (RN) brancos, os hemangiomas apresentam uma prevalência de 1,1 a $2,6 \%$ e é menos frequente em negros e japoneses $(0,8 \%)$. Segundo estudos nacionais, a incidência é de 3 a 4 casos, em cada 100 nascidos vivos. Observa-se marcada predominância pelo sexo feminino, uma tendência por tipos de pele clara e uma alta incidência em $\mathrm{RN}$ prematuros, especialmente os com peso inferior a $1500 \mathrm{~g}$. Mais da metade dos hemangiomas envolve o polo cefálico. A localização anatômica da lesão é o maior fator na determinação da probabilidade de complicações. ${ }^{1,6}$ As complicações mais comuns são: as ulcerações, ICC, hipotiroidismo, alteração da visão, comprometimento da audição e da respiração e desfiguramento.
Os hemangiomas podem ser classificados, clinicamente, em superficiais, profundos e mistos. ${ }^{5}$ Os hemangiomas superficiais estão localizados na derme superficial, os profundos na derme reticular e/ou subcutâneo e os mistos são dotados de um componente superficial e profundo (Figura 1). Apesar de a maioria dos casos necessitarem apenas de acompanhamento rigoroso, $15 \%$ necessitam de tratamento específico para minimizar futuras complicações. ${ }^{3}$ Existem várias modalidades terapêuticas descritas para tratamento dos hemangiomas e nenhuma delas se mostra totalmente eficaz para todos os casos (Figura 1).

Segundo a Academia Americana de Dermatologia, no manejo dos hemangiomas, as metas são: prevenir ou reverter as complicações dos hemangiomas alarmantes, prevenir o desfiguramento permanente, minimizar o estresse psicossial para pacientes e parentes, evitar procedimentos agressivos e potencialmente inestéticos e prevenir ou tratar adequadamente a ulceração minimizando as cicatrizes, infecções e dor. ${ }^{1}$

A conduta expectante ou não intervenção ativa sempre foi a mais utilizada, respeitando a história natural do hemangioma que é benigno $\mathrm{e}$ autolimitado. Lesões periorificiais, hemangiomas extensos (havendo risco de coagulopatia), ICC ou que possam causar deformidades devem ser tratados.

O corticoide sistêmico é o mais usado, principalmente nos casos graves. $O$ seu efeito principal é o de deter o crescimento e, se possível, induzir a regressão da lesão, possivelmente, por inibir a angiogênese e induzir a apoptose. ${ }^{8}$ A prednisona e a prednisolona são as drogas mais usadas por via oral, e a dose pode variar de 2 a $5 \mathrm{mg} / \mathrm{Kg} / \mathrm{dia}$. Os efeitos colaterais são bem descritos e geralmente transitórios. 9 Em pacientes com hemangioma periocular, a

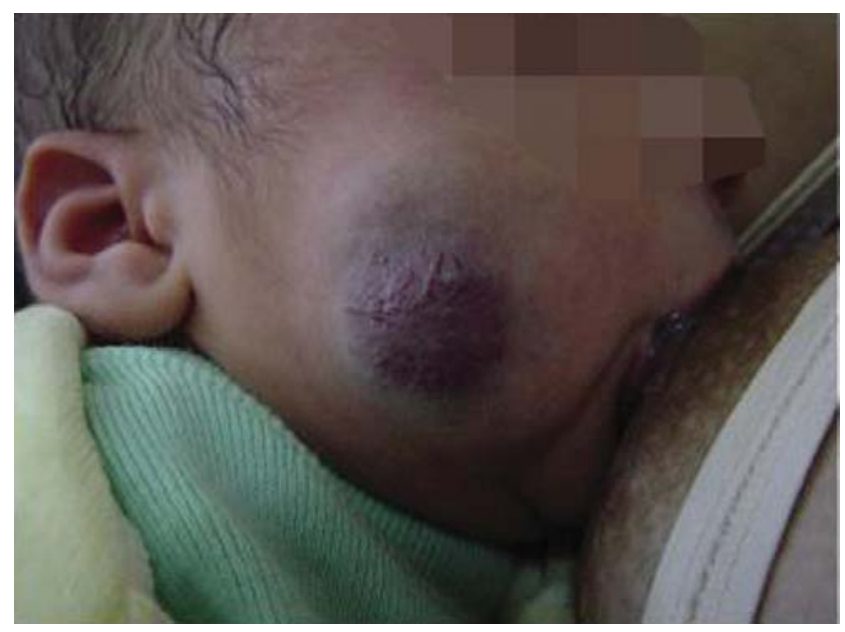

FigurA 1: Hemangioma misto em região geniana D 
pulsoterapia intravenosa com metilprednisolona mostrou diminuição significativa do risco de complicações oculares. ${ }^{10}$

O corticoide tópico ultrapotente tem sido testado, em hemangiomas superficiais, tanto que resultados mostraram-se satisfatórios. ${ }^{11}$ Geralmente, ao se ministrar o corticoide intralesional, a resposta é menor que a observada e efeitos colaterais também podem ocorrer. ${ }^{12}$

A corticoterapia intralesional é utilizada, principalmente, em hemangioma periocular ou lesões menores, mas seu uso é controverso, visto que há a possibilidade de efeitos colaterais graves. ${ }^{3,13}$

$\mathrm{O}$ uso do interferon está indicado para hemangiomas alarmantes, com risco de vida, não responsivos ao tratamento com corticoide oral. A resposta pode ser mais lenta que com o uso de corticoides; seu efeito angiogênico é eficaz com resposta de $50 \%$, com média de tratamento de 7 a 8 meses. ${ }^{9}$ Apresenta vários efeitos colaterais e risco de neurotoxicidade. ${ }^{14}$

Os agentes antineoplásicos têm sido usados, em casos raros, em que não há resposta ao tratamento com corticoides ou ao interferon. ${ }^{3}$ Há relato de boa resposta clínica, com efeitos colaterais moderados e transitórios, em menos de $50 \%$ dos casos.

A intervenção cirúrgica precoce é usada, em casos que possam afetar a autoestima dos pacientes, tais como: nos hemangiomas de ponta nasal, lesões pedunculadas e lesões de pálpebra, onde não respondem ao tratamento clínico. ${ }^{15}$ A técnica cirúrgica tradicional é a excisão lenticular, sendo a excisão circular uma técnica alternativa. ${ }^{16}$

A criocirurgia é mais efetiva, quando realizada em crianças maiores e em lesões pequenas. ${ }^{17}$ As complicações mais frequentes são: atrofia, cicatriz e alteração pigmentar. ${ }^{15}$

A escleroterapia é indicada para alguns hemangiomas profundos. Alguns agentes esclerosantes utilizados são: o etanol, álcool etílico, polidocanol, dentre outros. Complicações não são infrequentes e incluem necrose de pele e paralisia de nervo periférico. ${ }^{15,18}$

Novas terapêuticas têm sido testadas, dentre elas: o imunomodulador, de uso tópico em hemangiomas superficiais, na fase proliferativa, de tal forma que houve bons resultados. ${ }^{19,20}$

Diferentes tipos de laser podem ser usados, na abordagem do hemangioma, como: o argônio, $\mathrm{CO} 2$, Nd:YAG e mais recentimente o LPTDL (long-pulsed tunable dye laser $).{ }^{15}$

A embolização não é muito utilizada pelo risco de migração de partículas. Em tumores vasculares complicados, pode ser utilizada previamente à ressecção cirúrgica. ${ }^{15,21}$

A radioterapia está praticamente abandonada, por conta de suas sequelas, a longo prazo. ${ }^{13} \mathrm{~A}$ ligadura vascular é considerada, com reservas, nos casos de fístulas arteriovenosas e nos sangramentos intensos. ${ }^{6}$ A terapia compressiva é outra opção terapêutica. As desvantagens são: a possível ulceração e o desconforto da pressão local. ${ }^{15}$

O objetivo desse estudo foi analisar os diversos tratamentos utilizados para os hemangiomas nos pacientes pediátricos, atendidos no HUB, no período de março de 2000 a dezembro de 2006.

\section{MÉTODOS}

Foram incluídos, no estudo pacientes portadores de hemangiomas cutâneo ou cutâneomucoso, com idade entre 0 e 15 anos, atendidos nos ambulatórios de cirurgia pediátrica e de dermatologia pediátrica do Hospital Universitário de Brasília - HUB, de março de 2000 a dezembro de 2006.

$\mathrm{O}$ instrumento utilizado foi: a coleta de dados, a partir dos prontuários cadastrados pelo Serviço de Arquivo Médico e Estatístico do HUB e, a partir de questionário aplicado aos pais ou responsáveis, no ato da consulta, bem como pela avaliação clínica, laboratorial (se necessária) e fotográfica. O questionário abordava dados da identificação do paciente e perguntas relacionadas às intercorrências na gestação e parto, características clínicas do hemangioma, tipo de tratamento utilizado e avaliação do responsável e dos pesquisadores, em relação aos resultados pós-tratamento: cura total, parcial ou inalterado. A cura total foi definida como: regressão da lesão - acima de $80 \%$ em relação ao tamanho -, amolecimento ou clareamento. A cura parcial foi definida como regressão de 50 a $79 \%$ da lesão nesses mesmos parâmetros, e inalterado, quando a lesão manteve as características iniciais. Trata-se de corte clínica retrospectiva analítica, tendo como variáveis os tipos de tratamento (variável independente) e os resultados (variável dependente: cura total, parcial e inalterada).

Para análise dos dados, empregou-se o Statystical Package for Social Science, versão 13.0; 2004. Para pesquisar a associação entre as variáveis estudadas, utilizou-se o teste c2 e razão de chance (odds ratio), considerando como significante uma relação de $\mathrm{p}<0,05$.

\section{RESULTADOS}

Foram analisados 122 casos, sendo $60,7 \%$ do sexo feminino (74/122) e 39,3\% do masculino (48/122), apresentando uma razão de sexo (F: M) de 1,5: 1.

13,9 \% das mães $(17 / 122)$ relataram a presença de fatores pré-natais, como ameaça de aborto e/ou sangramento, no primeiro trimestre da gestação. A prematuridade acometeu $14,8 \%$ dos pacientes estudados (18/122), em oposição a 85,2\% (104/122), em que tiveram seu nascimento a termo. A razão de RN pré-termo e $\mathrm{RN}$ a termo é de 1:5, 7 .

Dos 122 pacientes, $101(82,8 \%)$ apresentavam 
lesões precursoras ou hemangiomas iniciais, logo após o nascimento (até 30 dias), e em 21 pacientes $(17,2 \%)$, os pais observaram as lesões, após quatro semanas do nascimento. O crescimento da lesão foi observado, em 103 pacientes $(84,4 \%)$ e em 19 $(15,6 \%)$, portanto as lesões se mantiveram inalteradas. Em 111 pacientes (91\%) e as múltiplas em 11 pacientes (9\%), as lesões isoladas ocorreram.

42 casos $(34,4 \%)$ foram classificados como hemangiomas superficiais, $13(10,7 \%)$ como profundos e 67 (54,9\%) como lesões mistas. Em relação à localização, 79 localizavam-se no polo cefálico $(64,8 \%), 23$ em tronco $(18,9 \%), 18$ em membros (14,8\%) e oito no períneo (6,6\%). Foram padronizadas como: pequena (as lesões menores que $3 \mathrm{~cm}$ ), média (as lesões entre 3 e $5 \mathrm{~cm}$ ) e grande (as maiores que $5 \mathrm{~cm}$, no seu maior diâmetro). Os hemangiomas pequenos ocorreram em 64 pacientes (52,5\%), os médios em 33 (27\%) e os grandes em 25 pacientes (20,5\%). A ulceração e o sangramento estavam presentes em 12 pacientes $(9,8 \%)$. A associação de hemangiomas com síndromes ocorreu em sete pacientes (5,7\%), sendo elas: as Síndromes PHACES, Sturge weber, Dandy Walker e Síndrome Kabasach-Merritt.

98/122 dos pacientes foram submetidos a tratamento único e 24 necessitaram de tratamentos múltiplos. No grupo de tratamento único, as terapêuticas utilizadas foram: conduta expectante, em 38 pacientes (38,8\%); compressão, em 3 casos $(3,1 \%)$; corticoide sistêmico, em 18 pacientes $(18,4 \%)$; corticoide intralesional, em 13 pacientes $(13,3 \%)$; corticoide tópico, em 4 casos $(4,1 \%)$; cirurgia convencional, em 12 pacientes $(12,2 \%)$; criocirurgia, em 7 pacientes (7,1\%); laser e/ou luz pulsada, em 1 paciente (1\%); imiquimod, em 2 pacientes $(2 \%)$.

Dos 24 pacientes, dos quais foram submetidos a tratamentos variados, 15 fizeram 2 tipos de tratamentos e 9 necessitaram de 3 ou mais tratamentos. 7 pacientes fizeram uso de corticoide intralesional e posterior cirurgia convencional (29,2\%), 6 pacientes usaram o corticoide oral, seguido de cirurgia convencional (25\%), 1 paciente fez uso de corticoide oral e intralesional (4,2\%), 1 paciente utilizou corticoide tópico e criocirurgia $(4,2 \%)$ e 9 pacientes necessitaram de 3 ou mais tratamentos (37,5\%). Nesse grupo, as lesões localizavam-se, com maior frequência, no polo cefálico.

Em 36 pacientes (29,5\%), com relação ao tratamento, a cura total foi relatada pelos pais, já em 78 casos $(63,9 \%)$, cura parcial e em 8 pacientes $(6,6 \%)$, não houve alteração da lesão com o tratamento.

Os 8 pacientes, que apresentaram resposta inalterada, foram conduzidos com o tratamento expectante e diagnosticados, posteriormente, como mancha vinho do porto. Dessa forma, esses pacientes não foram considerados para cruzamento dos dados.

Assim, dos 90 pacientes submetidos a tratamento único para hemangioma, 28 apresentaram cura total $(31,2 \%)$ e 62 cura parcial $(68,8 \%)$.

Destes, 30 obtiveram conduta expectante, 5 apresentaram cura total $(16,7 \%)$ e 25 tiveram cura parcial (83,3\%) (Figura 2).

Em 3 pacientes, utilizou-se a compressão, em que 1 apresentou cura total $(33,3 \%)$ e 2 cura parcial (66,7\%) (Figura 3).

Em 18 pacientes, administrou-se o corticoide oral, assim, ocorrendo cura total em 5 pacientes $(27,8 \%)$ e parcial em 13 (72,2\%) (Figura 4).

Em 13 pacientes, aplicou-se o corticoide intralesional e se observou cura total em 1 caso (7,7\%), e parcial em 12 pacientes (92,3\%) (Figura 5).

O corticoide tópico foi utilizado em 4 pacientes, sendo que 3 obtiveram cura parcial e 1 cura total (Figura 6).

Em 12 pacientes, realizou-se a cirurgia convencional, de maneira que todos apresentaram cura total das lesões e sem recidiva do hemangioma
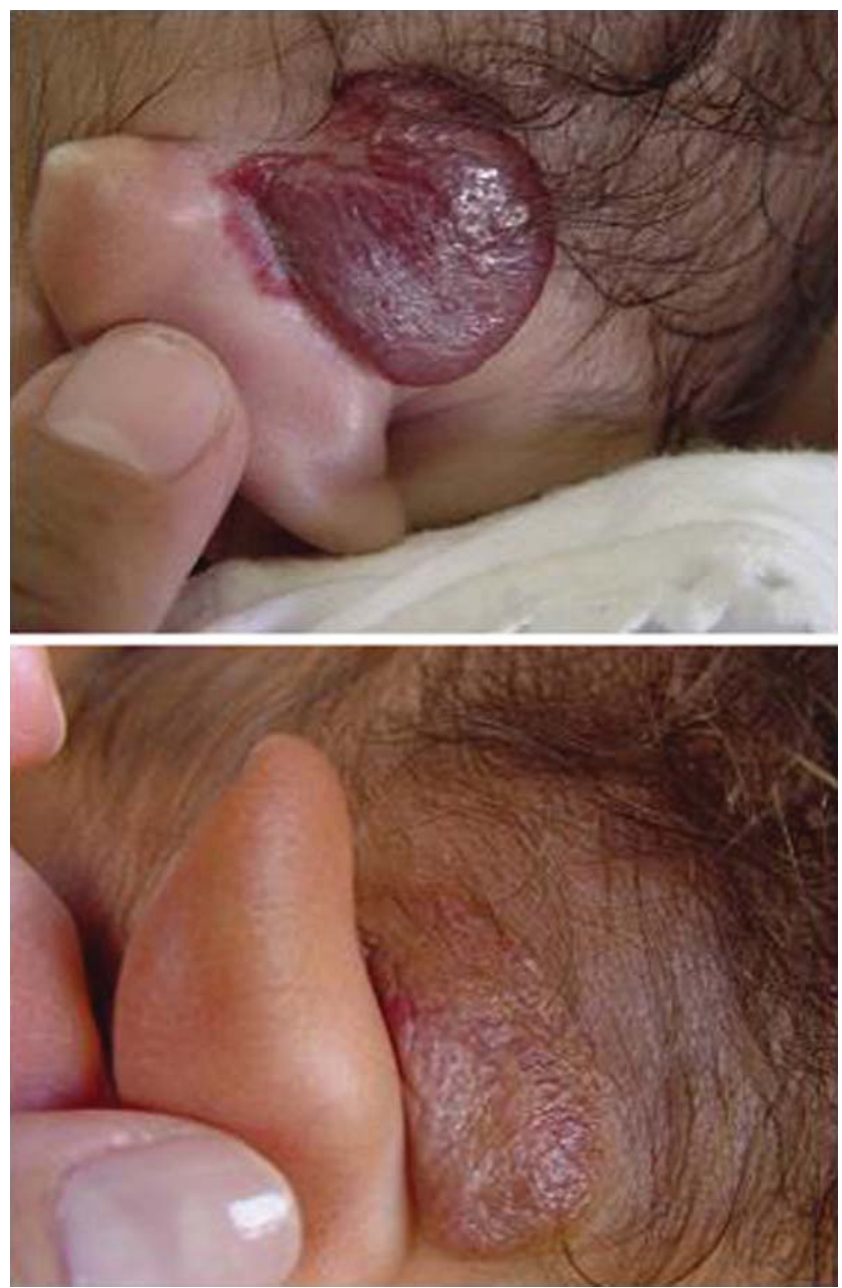

Figura 2: Tratamento com conduta expectante. Hemangioma retroauricular à E. Involução da lesão, em 22 meses, com cura total 

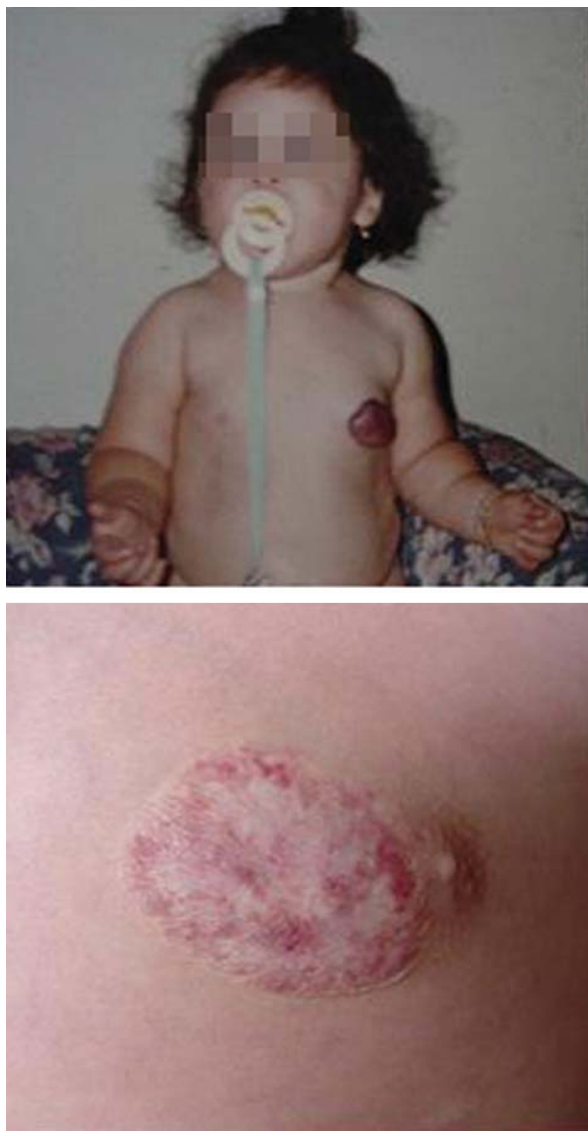

Figura 3:

Tratamento com compressão. Hemangioma em mama E, com cura total

nas bordas da cicatriz (Figura 7).

Realizou-se a criocirurgia em 7 pacientes: 5 apresentaram cura parcial e 2 cura total (Figura 8).

1 paciente foi submetido à luz pulsada, com cura parcial, e interrompeu o tratamento, pois sinalizaram dor.

O imiquimod de uso tópico foi utilizado em 2 pacientes com boa resposta: um com cura total e o outro com cura parcial (Figura 9).

Dos 24 pacientes submetidos a tratamentos múltiplos, 12 (50\%) tiveram cura total e 12 (50\%) pacientes obtiveram cura parcial. 7 foram submetidos, inicialmente, à corticoterapia intralesional e, posteriormente, à cirurgia convencional, sendo que 5 obtiveram cura total $(71,4 \%)$ e 2 cura parcial $(28,6 \%)$. 6 pacientes utilizaram corticoide oral e, depois, submetidos à cirurgia convencional; 4 obtiveram cura total $(66,7 \%)$ e 2 cura parcial (33,3\%) (Figura 10). 1 paciente utilizou corticoide oral e intralesional, com cura total e 1 paciente utilizou corticoide tópico e criocirurgia, com cura parcial.

9 pacientes necessitaram de 3 ou mais tratamentos; 2 tiveram cura total $(22,2 \%)$ e 7 , cura parcial $(77,8 \%)$.

O cruzamento das variáveis modalidades terapêuticas utilizadas e resultados, após o tratamento, não apresentou concordância significativa. A conduta expectante relacionada com cura total e parcial apresentou um OR 2,37 , porém com IC $(0,769$ 6,986) e p 0,129, não mostrando relação de causalidade nesta amostra, o que aconteceu também com as condutas de compressão, corticoide sistêmico, corticoide intralesional, corticoide tópico, criocirurgia e imiquimod. A limitação do tamanho da amostra dos pacientes com tratamento único $(\mathrm{n}=90)$ afetou os resultados, pois os mesmos foram distribuídos entre os tipos de tratamento, resultando em um n pequeno para cada modalidade terapêutica. Para o tratamento laser/Luz pulsada, não foi possível calcular o OR, p e o intervalo de confiança, pois só havia um paciente com cura parcial da lesão (100\% de cura parcial). A avaliação da cirurgia convencional foi estatisticamente significativa, com $\mathrm{p}$ próximo à zero, porém, sem intervalo de confiança, pois todos os casos apresentaram cura total (percentual de 100\% de cura).

\section{DISCUSSÃO}

Neste estudo, obteve-se uma razão de sexo (F:M) de 1,54:1. A predominância dos hemangiomas, no sexo feminino, relatada na literatura foi observada no corpus, porém em proporção menor. ${ }^{1,3,6}$
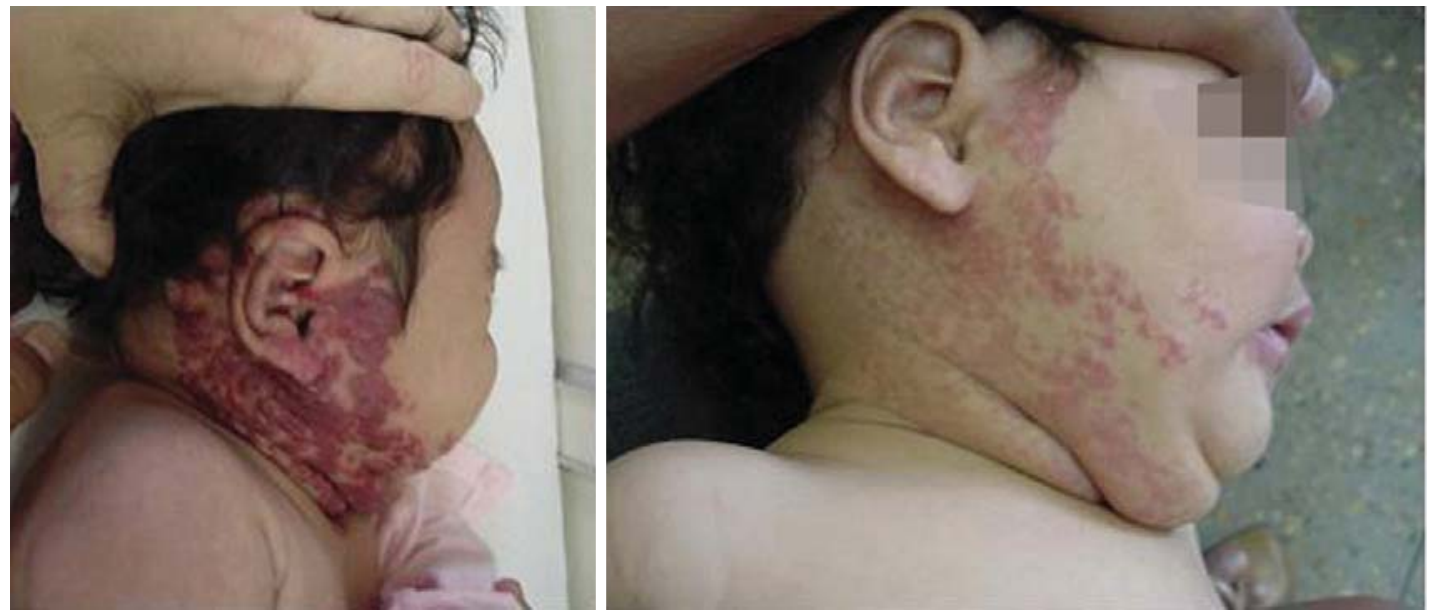

Figura 4: Tratamento com corticoide oral. Hemangioma em região de "barba".

Regressão da lesão, no período de seis meses com cura total 

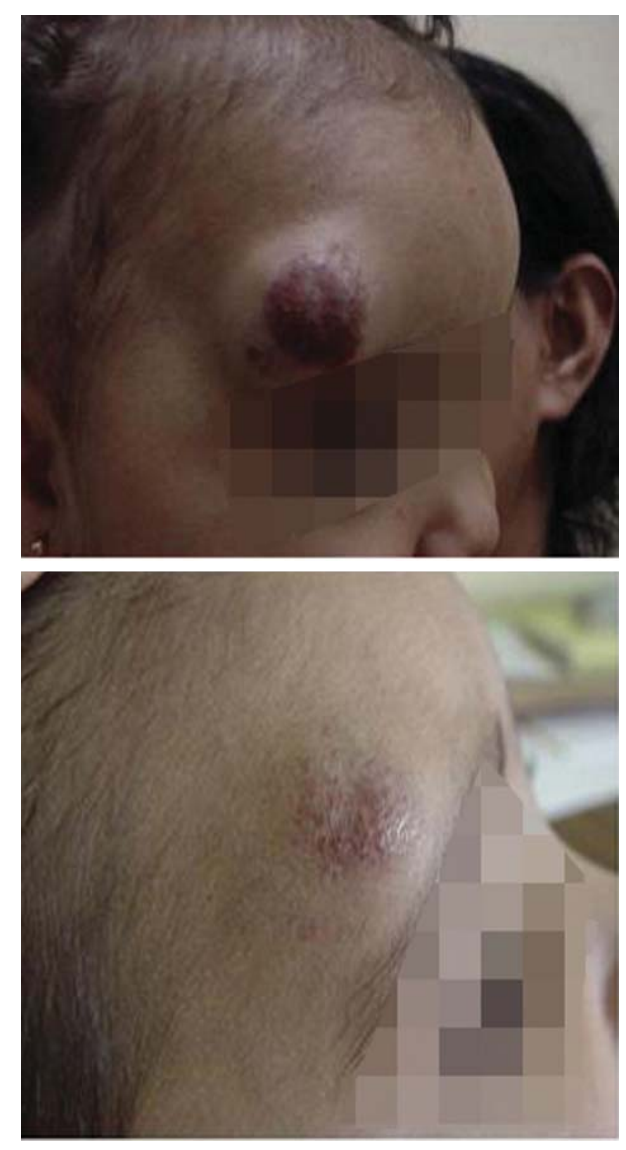

Figura 5: Tratamento com corticoide intralesional. Hemangioma, em região frontal $\mathrm{D}$. Regressão da lesão, após quatro aplicações da medicação, com cura total

Apesar da significativa associação de procedimentos invasivos durante a gestação, como a punção de vilosidade coriônica, com o surgimento de hemangioma, ${ }^{22}$ esse dado não foi avaliado neste trabalho, visto que o grupo em estudo não tem acesso a este tipo de procedimento.

O dado relacionado com ameaça de aborto e/ou sangramento, no primeiro trimestre da gestação, é um fator importante, por conta do risco de descolamento placentário parcial e a uma provável associação com hemangioma. No estudo atual, 17 pacientes $(13,9 \%)$ relataram este dado positivo.

A predominância dos hemangiomas nos prematuros é bem documentada, sendo observado em 23 a $30 \%$ de RN prematuros, com peso menor que $1.000 \mathrm{~g}$, e $15 \%$ em $\mathrm{RN}$, com peso entre 1000 a $1500 \mathrm{~g}$. A razão de $\mathrm{RNPT} / \mathrm{RNT}$ é de $4: 1 \mathrm{em}$ prematuros $<1 \mathrm{~kg}$, conforme preconiza a literatura. ${ }^{1,23}$ Nesta casuística, 18 pacientes $(14,8 \%)$ relataram prematuridade, com uma razão de $\mathrm{RN}$ pré-termo e $\mathrm{RN}$ a termo de 1: 5,7, dado inverso ao que é apontado pela literatura internacional, porém GOLDENBERG e cols (2001) relatam 95,8\% dos pacientes, nascidos a termo. ${ }^{24}$ Nos hospitais assistenciais da rede pública, a baixa sobrevida de RN extremamente prematuros $(<1000$ g) colabora para estes dados.
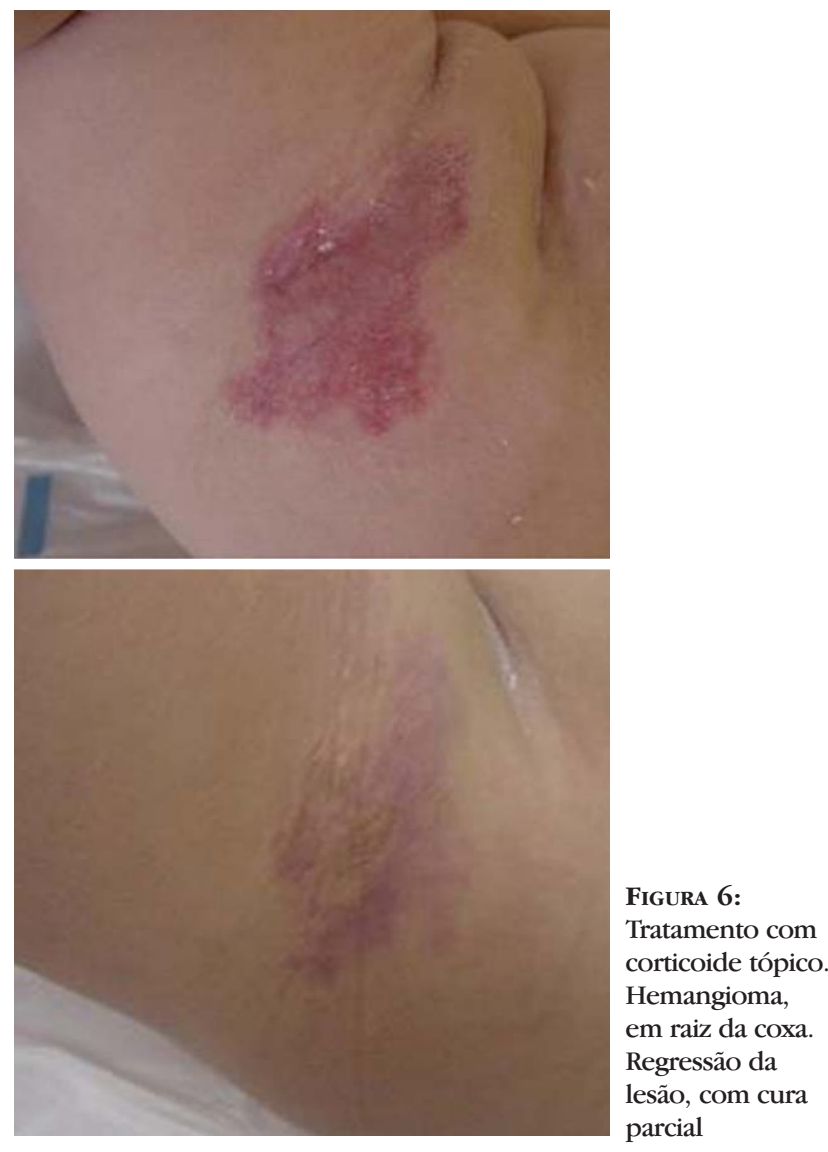

$\mathrm{Na}$ literatura internacional, as lesões precursoras ocorrem em 30 a $50 \%$ dos pacientes ${ }^{3}$, o aparecimento da lesão, antes de um mês de idade, ocorreu de $77 \%$ a $88,7 \%$ dos casos ${ }^{6,25}$, e a incidência de hemangiomas ou sinais precursores ao nascimento é de $56,8 \%{ }^{24}$. No presente estudo, encontrou-se um percentual mais elevado de lesões, antes de um mês de vida $(82,8 \%)$. Esta casuística maior, em relação às lesões precursoras, pode estar associada ao fato de a pesquisa ter sido realizada em hospital de referência, porque recebe casos de maior complexidade.

O crescimento da lesão, após o nascimento, ocorreu em 103 pacientes (84,4\%) e em 19 (15,6\%), as lesões se mantiveram inalteradas. Segundo CAMPOS (2000), o crescimento rápido da lesão foi observado em $89,5 \%$ dos casos. ${ }^{6}$

As lesões isoladas ocorreram em 111 pacientes (91\%) e as múltiplas em 11 pacientes (9\%), o que converge com a literatura, segundo a qual relata a ocorrência da forma isolada em 80 a $85 \%$ dos pacientes e lesões múltiplas em 15 a 30\%. ${ }^{1,3,25}$

Constatou-se predomínio de hemangiomas mistos. Dos 122 pacientes, 34,4\% apresentavam hemangiomas superficiais, 54,9\%, lesões mistas e $10,7 \%$ lesões profundas. Esses dados divergem com os dados da literatura mundial, uma vez que relata lesões 

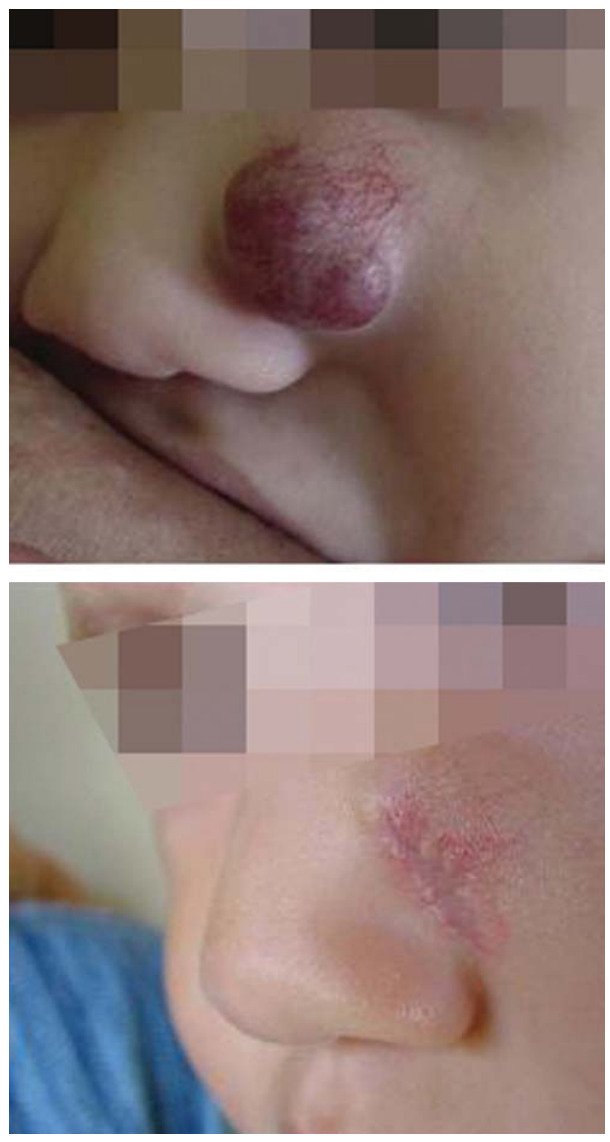

FIGURA 7:

Tratamento

cirurgia

convencional.

Hemangioma

nasal à $\mathrm{E}$.

Regressão da

lesão, com

cura total

superficiais, com percentual de 50 a $60 \%$, lesões mistas de 25 a $35 \%$ e lesões profundas em 15\% dos pacientes. ${ }^{23} \mathrm{Na}$ literatura nacional, observou-se o predomínio de lesões superficiais: 95,8\%. 6 GOLDENBERG (2001) relata 34,1\% lesões superficiais, $27,3 \%$ profundas e $38,6 \%$ lesões mistas. DUTRA (2003) relata 63,9\% de lesões superficiais/mistas e $11,2 \%$ de lesões profundas. ${ }^{6,24,26}$ Esta divergência de dados se deve ao fato de a maioria das lesões superficiais involuir espontaneamente, de forma mais breve, não sendo encaminhadas para hospitais de referência como o HUB.

A avaliação da localização dos hemangiomas demonstrou que 79 estavam localizados no polo cefálico $(64,8 \%), 23 \mathrm{em}$ tronco $(18,9 \%), 18 \mathrm{em}$ membros $(14,8 \%)$ e 8 no períneo $(6,6 \%)$. Alguns pacientes apresentavam múltiplos hemangiomas. Os dados encontrados nesse estudo coincidem com os da literatura, segundo a qual relata, em $60 \%$ dos pacientes, a ocorrência dos hemangiomas em polo cefálico, $25 \%$ em tronco e $15 \%$ nas extremidades. ${ }^{1}$ Autores brasileiros observaram $71,6 \%$ no pólo cefálico, $20,3 \%$ em tronco, $20 \%$ em membros, $4,3 \%$ no períneo e $0,7 \%$ em vísceras. ${ }^{6}$

Os hemangiomas pequenos $(<3 \mathrm{~cm})$ foram observados em 64 pacientes $(52,5 \%)$, as lesões médias
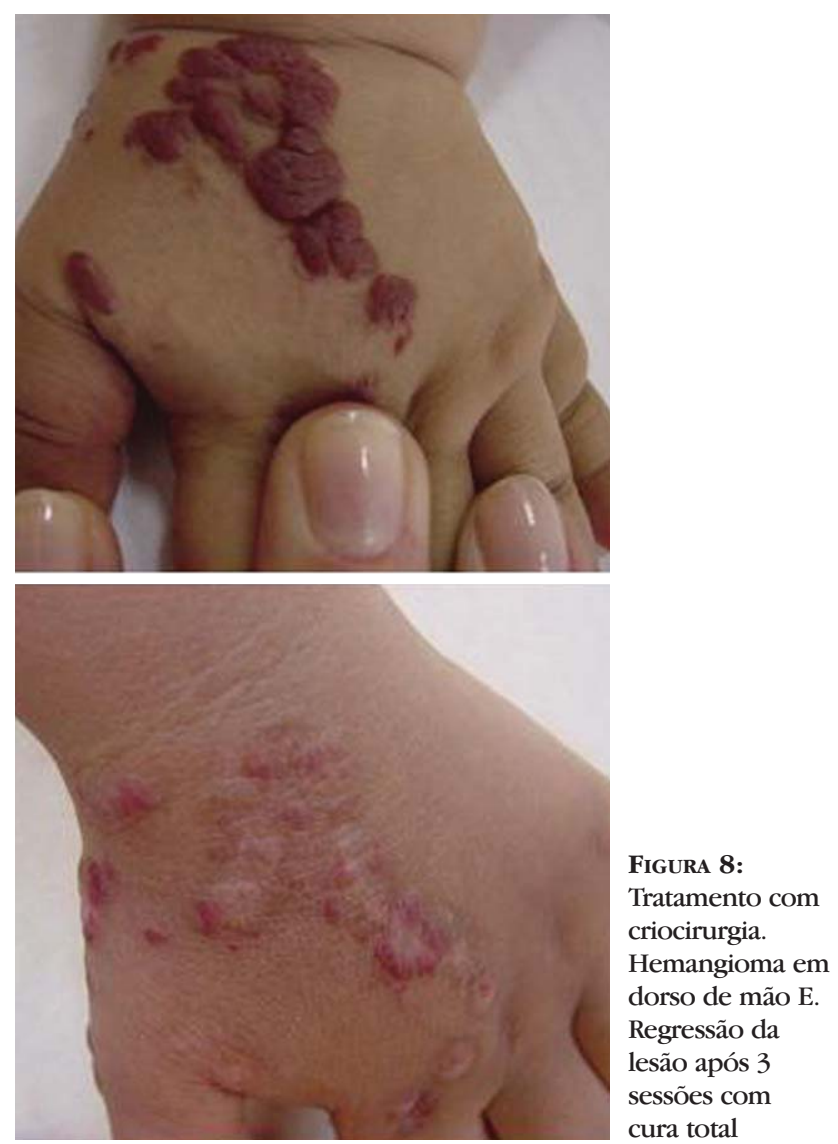

(entre 3 e $5 \mathrm{~cm}$ ) em 33 pacientes (27\%) e os hemangiomas grandes $(>5 \mathrm{~cm})$ em 25 pacientes $(20,5 \%)$. Na literatura internacional, não há dados avaliando o tamanho das lesões, porém, na literatura nacional, trabalho realizado por CAMPOS (2000), relata $64 \%$ das lesões $<5 \mathrm{~cm} \mathrm{e} 35,2 \%$ das lesões $>5 \mathrm{~cm} .{ }^{6}$

A ulceração é a complicação mais frequente, já que incidem em 5 a $13 \%$ dos casos, segundo a literatura internacional. ${ }^{7,23}$ A literatura nacional relata ocorrência de ulceração em 35,9\% dos casos e 22,6\% de sangramento, em pacientes com hemangiomas. 6 Em 9,8\% dos pacientes avaliados, a casuística apresentada apontou ulceração e sangramento, convergindo com os dados da literatura mundial.

Em 5,7\% dos pacientes, o estudo constatou que há associação de hemangiomas com síndromes, corroborada por CAMPOS (2000), no qual relata que as síndromes hemangiomatosas ocorreram, em 4,9\% dos casos. ${ }^{6}$ Estudo realizado por DUTRA (2003) com pacientes portadores de anomalias vasculares da região orbitária, num período de 15 anos, constatou que em 8/169 dos pacientes avaliados ocorre a associação de síndromes (PHACES, Dandy-Walker, Kasabach-Merritt e Proteus). ${ }^{26}$

Os dados desse estudo convergem com os dados relatados na literatura, referentes aos fatores de 

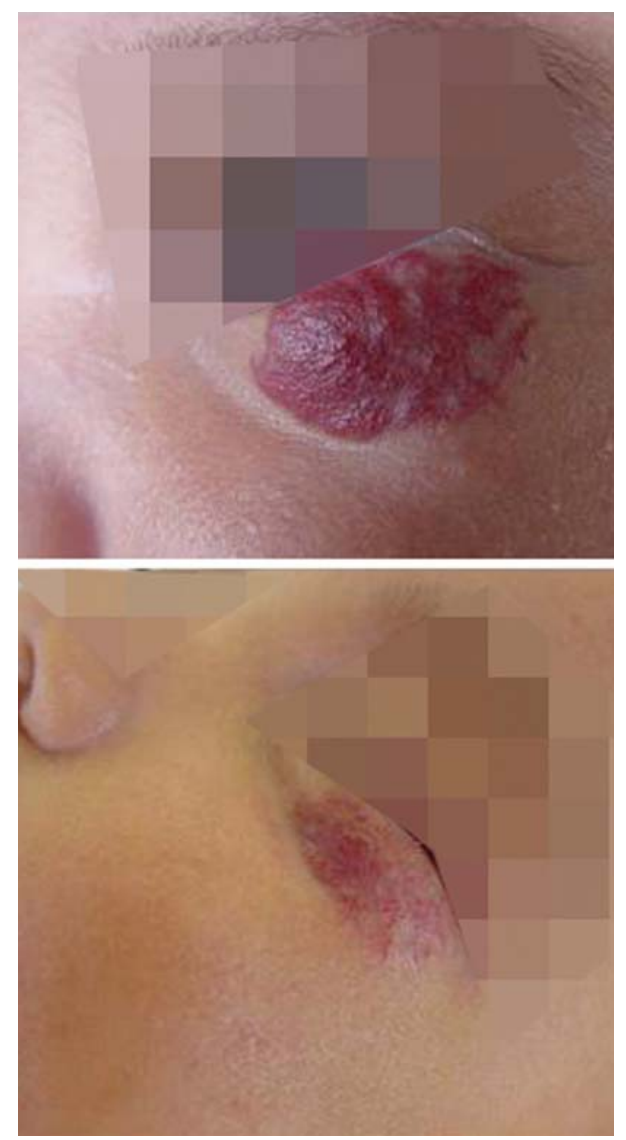

FIGURA 9:

Tratamento com imiquimod. Hemangioma, em pálpebra inferior $\mathrm{E}$ Regressão da lesão, após 2 meses $(2 \mathrm{x}$ por semana por 5 semanas), com cura total

gênero, pré-natais e perinatais, características como: presença ao nascimento, crescimento, número de lesões. Não foi observada associação entre as variáveis pré-natais, perinatais, presença de ulceração e sangramento, presença de síndromes associadas e os resultados pós-tratamento.

No grupo de pacientes que utilizou apenas 1 tratamento $(\mathrm{n}=98), \quad 38,8 \%$ dos pacientes apresentaram conduta expectante e 61,2\% necessitaram de alguma intervenção terapêutica medicamentosa ou cirúrgica. A literatura internacional relata que apenas 10 a $20 \%$ dos hemangiomas necessitam de tratamento clínico ou cirúrgico, sendo a conduta expectante adotada nos $80 \%$ restantes. $^{3}$

Dos 122 pacientes: 29,5\% dos pacientes alcançaram a cura total; $63,9 \%$ dos pacientes, cura parcial e 6,6\% não apresentaram resposta ao tratamento. Segundo DUTRA (2003), em 59,5\% dos pacientes, a cura total foi observada e cura parcial, em $40,8 \%{ }^{8}$ CAMPOS (2000) relata cura total e/ou parcial em $84,3 \%$ e a ausência de resposta ao tratamento foi observada em $13,4 \%$ do seu estudo. ${ }^{6}$

No presente estudo, $38,8 \%$ dos pacientes apresentaram conduta expectante, coincidindo com estudos, nos quais ocorreu em 34,9 a $36,4 \%$ dos casos. ${ }^{24,26}$ DELLA NINA e cols (2006) referem conduta
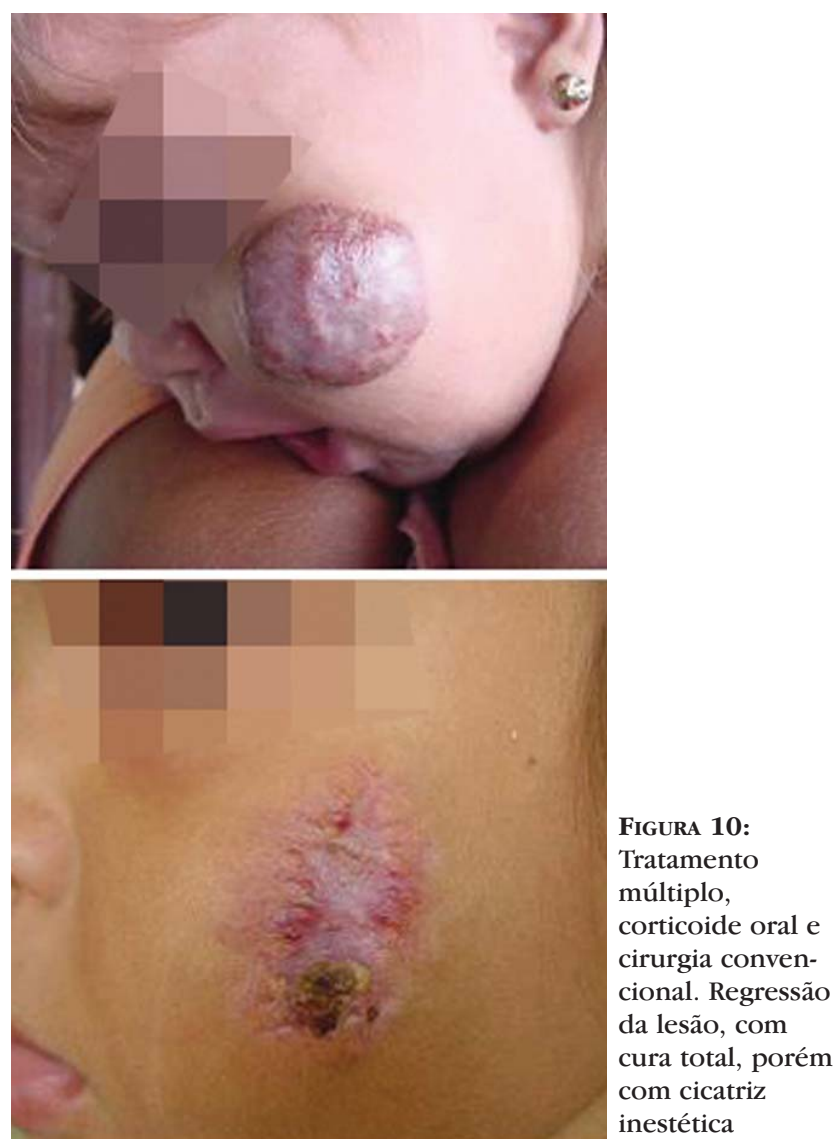

expectante em $75 \%$ dos casos. $57,5 \%$ em lesões superficiais e $17,5 \%$ em lesões profundas, com cura total em $40 \%$ do pacientes, com conduta expectante.

${ }^{25}$ A divergência no percentual de conduta expectante, em relação à literatura mundial, se deve ao viés de que os pacientes, da presente amostra, não traduzem a realidade da população geral, pois foram referenciados para atendimento terciário, porque, de forma geral, estas formas mais complexas da patologia necessitam de intervenção clínica ou cirúrgica.

Entre os pacientes (5/30), com conduta expectante, observou-se um menor número de casos, com cura total, provavelmente, em função de as lesões ainda estarem em estado de regressão. Estimase que, até 9 anos, 90\% dos hemangiomas apresentem regressão. ${ }^{13}$ Naqueles pacientes, em que se constatou a cura total, apresentavam lesões pequenas e, a maioria, superficiais, com involução total da lesão.

Em estudo retrospectivo realizado com 543 pacientes com hemangiomas, observou-se que 15,8\% dos pacientes utilizaram tratamento sistêmico (corticoide ou interferon) e 17,3\% foram submetidos à cirurgia convencional. ${ }^{6}$ No presente estudo, $22,1 \%$ dos casos utilizou corticoide oral com cura total em $27,8 \%$ dos pacientes. DELLA NINA e cols (2006) relatam 17,5\% de pacientes tratados com corticoide oral. O corticoide 
oral é a terapia de $1^{\mathrm{a}}$ escolha para hemangiomas, com risco de complicações ou potencial de desfiguramento. ${ }^{27}$ DUTRA (2003) refere o tratamento clínico sistêmico, em $21,9 \%$ e intervenção cirúrgica pós-tratamento clínico, em $20,7 \%$ dos pacientes. ${ }^{26}$

$\mathrm{O}$ uso de corticoide intralesional (CIL para hemangiomas) é controverso pelo risco de efeitos colaterais. ${ }^{3} \mathrm{Na}$ casuística apresentada, 13 pacientes $(13,3 \%)$ foram submetidos a este tratamento, com cura parcial, em 92,3\% dos casos, na maioria lesões profundas ou mistas, e $7,7 \%$, de cura total das lesões. Os pacientes foram submetidos a 3 ou 4 aplicações, com intervalo de 4 a 6 semanas. Uma paciente apresentou equimose, após a aplicação com resolução espontânea. Segundo CHEN e cols (2000), 85\% dos pacientes tratados com CIL apresentaram uma redução de $50 \%$ do volume da lesão.

$\mathrm{Na}$ literatura, o uso da corticoterapia tópica também é controverso e alguns autores relatam efeitos colaterais importantes e eficácia menor, quando comparado ao corticoide intralesional. ${ }^{12}$ No presente estudo, em 4 pacientes (4,1\%), o corticoide tópico foi utilizado; 3 obtiveram cura parcial das lesões e 1 obteve cura total. 3 pacientes apresentaram ulceração, com sangramento em lesões localizadas no períneo, e todos utilizaram a medicação uma vez por dia, por três semanas, muitas vezes, associados à pomada cicatrizante. Estudo recente da literatura relata uma boa resposta com corticoide tópico em $74 \%$ dos pacientes. ${ }^{11}$

Em 3 pacientes (3,1\%), realizou-se a compressão, obtendo-se a cura parcial de $66 \%$ e total de $33,3 \%$, provavelmente, por conta do componente profundo da lesão, mas 1 caso de lesão mista evoluiu para cura total. Na literatura, a terapia compressiva é mais utilizada para lesões vasculares em extremidades. ${ }^{15}$

Nessa casuística, para 7 pacientes $(7,1 \%)$, utilizou-se a criocirurgia e 5 apresentaram cura parcial. Um paciente (com lesão em grande lábio) e outro (com lesão em extremidade) obtiveram resposta de cura total, com discreta hipocromia transitória. Os pacientes foram submetidos a 2 ou 3 sessões, com técnica de spray ou de contato, e tempo de congelamento da lesão de 10 a 30 segundos. DELLA NINA e cols (2006) relatam criocirurgia em 7,5\% dos pacientes. ${ }^{25}$ Estudos relatam eficácia em lesões superficiais próximas a do PDL. ${ }^{15,17}$

Aplicou-se, em 1 paciente, totalizando 3 sessões, a técnica de luz intensa pulsada, com intervalo de quatro semanas. A paciente abandonou $o$ tratamento, pois se queixou de dor e relatou cura parcial. Nenhum paciente fez uso de laserterapia, pois este recurso não estava disponível no HUB. Na literatura, o uso de laser e luz pulsada para lesões superficiais possui um sucesso considerável. ${ }^{29}$
O uso de imiquimod é uma opção terapêutica promissora, principalmente, para lesões de pequena e média dimensão e com componente profundo menor. ${ }^{19,20}$ Os dois pacientes (2\%) tratados com esta medicação obtiveram resposta rápida, obtendo-se a cura parcial do paciente, com lesão paranasal, e cura total do paciente, com lesão em pálpebra inferior. Efeitos colaterais locais como: inflamação e eritema são relatados pela literatura e também observados nesse estudo, tanto que foi necessária a suspensão da medicação por 15 dias. Estudo realizado por $\mathrm{HO}$ e cols (2007) relata cura total em 4/18 pacientes, com hemangiomas superficiais, e cura parcial, dos hemangiomas mistos, demonstrando uma diferença estatística significativa entre os resultados das lesões superficiais em relação às lesões misto-profundas. ${ }^{30}$

A cirurgia convencional é realizada, geralmente, em lesões pedunculadas, lesões em pálpebra, nariz, periorificiais ou lesões as quais não respondam ao tratamento clínico preconizado. ${ }^{11}$ No presente estudo, 12 pacientes $(12,2 \%)$ submetidos a tratamento único $(\mathrm{n}=98)$, foram indicados à intervenção cirúrgica e obtiveram cura total das lesões. Estudos relatam a cirurgia convencional para o tratamento de 17,3 e $27,2 \%$ dos pacientes, diagnosticados com hemangioma. ${ }^{2,6}$ No presente estudo, $16,3 \%$ de todos os pacientes $(n=122)$ foi submetido à cirurgia convencional. DUTRA (2003) relata intervenção cirúrgica pós-tratamento clínico, em $20,7 \%$,e cirurgia convencional, em $22,2 \%$ dos pacientes. ${ }^{26}$

$\mathrm{O}$ hemangioma em ponta nasal é um desafio. $\mathrm{O}$ tratamento de escolha para estes casos é, habitualmente, a cirurgia convencional. No presente estudo, 4 casos de hemangioma nasal foram analisados; submetidos 2 pacientes à cirurgia convencional, o que denotou a cura total, o $3^{\circ}$ paciente foi submetido à corticoide oral e o $4^{\circ}$ submetido à corticoide intralesional, indicando, assim, boa resposta, nos dois últimos casos (cura parcial).

No grupo de pacientes submetidos a múltiplos tratamentos $(n=24), 15(62,5 \%)$ necessitaram de 2 tipos de tratamento e em 9 pacientes $(37,5 \%)$, foram necessários 3 ou mais modalidades terapêuticas. No primeiro subgrupo dos tratamentos múltiplos, estas modalidades foram: cirurgia convencional associada à infiltração de corticoide intralesional, cirurgia convencional associada à corticoterapia oral, corticoide oral associado à infiltração de corticoide intralesional e corticoide tópico associado à criocirurgia.

A cirurgia convencional sempre ocorreu, após o primeiro tratamento, com exceção de um paciente. $\mathrm{O}$ subgrupo, com três ou mais tratamentos, apresentou grande variedade de combinação de tratamentos. Vale relatar que, destes 9 pacientes, 8 foram submetidos à cirurgia convencional e todos utilizaram corticoide 
oral. Os pacientes, que foram submetidos a dois ou mais tratamentos, geralmente, apresentavam lesões mais complexas ou a preocupação excessiva dos pais induziram à associação de mais tratamentos.

Nesta amostra, durante o período estimado, outros tratamentos como: embolização, escleroterapia, drogas antineoplásicas e interferon não foram utilizadas.

Este trabalho reforça as informações existentes na literatura, em relação ao sexo, características clínicas das lesões e resultados póstratamento e mostra diferenças relacionadas com o percentual de prematuridade e de lesões presentes ao nascimento. A ameaça de aborto e/ou sangramento, no primeiro trimestre da gravidez, é um dado novo não relatado na literatura, tendo associação aos hemangiomas, assim como outros estudos comprovaram a maior incidência dos hemangiomas, em filhos de mães submetidas à punção de vilosidade coriônica, no primeiro trimestre da gestação.

O seguimento de 7 anos contribuiu com dados pouco relatados na literatura, como: ameaça de aborto na gravidez, maior número de lesões presentes - logo após o nascimento - e uma maior predominância de lesões mistas. Aproximadamente, $1 / 3$ dos pacientes apresentaram conduta expectante acompanhando a involução natural do hemangioma. A conduta expectante continua sendo a medida mais adotada por muitos estudiosos do assunto. As intervenções ocorrem, na maioria dos casos complexos, por conta de os riscos anatômicos e ou funcionais que podem acontecer. $\mathrm{Na}$ população infantil, é muito importante que as medidas adotadas sejam definitivas e eficazes, no sentido de diminuir a morbidade, do ponto de vista psíquico, anatômico e funcional destas crianças. A abordagem individualizada para cada paciente é imprescindível, para um resultado final satisfatório, minimizando as sequelas anatômicas e funcionais bem como reduzindo o impacto da patologia, na autoestima do paciente e no convívio social dos familiares.

O presente trabalho não é o modelo de estudo mais adequado para avaliar a relação dos vários tipos de tratamentos empregados, em relação à cura, porém é o ponto de partida para que outros estudos contribuam na avaliação de tratamentos mais adequados para os hemangiomas da infância.

\section{CONCLUSÕES}

O manejo dos hemangiomas é difícil e controverso, com espectro clínico amplo e severidade variada. Soma-se a falta de estudos prospectivos, relacionados aos tratamentos, com estudos baseados em evidência.

Este estudo é superponível à literatura, em relação às variáveis de sexo, localização, número, dimensões das lesões, presença de ulceração e associação com síndromes.

Observou-se uma discordância com a literatura, em relação ao percentual de lesões presentes ao nascimento e de lesões mistas, estas foram maiores, comparativamente, aos dados da literatura.

Neste corpus, Os fatores pré e perinatais não apresentaram relação com hemangioma e a prematuridade não foi expressiva.

A análise das modalidades terapêuticas empregadas não demonstrou concordância significativa entre os tratamentos utilizados e os resultados, com exceção da cirurgia convencional, que obteve os melhores resultados, em relação à cura do hemangioma.

A criocirurgia e o imiquimod demonstraram uma boa resposta terapêutica.

A cura parcial também traduz uma melhora das lesões do ponto de vista funcional, anatômico e estético. Quando algumas lesões foram avaliadas, certificou-se, neste trabalho, sua cura parcial, que tendem à involução total, demonstrando, sobretudo, a eficácia dos tratamentos e confirma os bons resultados obtidos nesta pesquisa.

Quanto ao tratamento mais adequado, torna-se de variável, de caso a caso, e deve ser avaliado dentro de vários contextos: identificando os hemangiomas que necessitam de tratamento, o momento mais adequado para a intervenção, as implicações psicossociais do tumor sobre a criança e seus familiares e a melhor opção terapêutica, analisando sempre o risco benefício do tratamento. 


\section{REFERÊNCIAS}

1. Frieden IJ, Eichenfield LF, Esterly NB, Geronemus R, Mallory S. Guidelines of care for hemangiomas of infancy. American Academy of Dermatology Guidelines/Outcomes Committee. J Am Acad Dermatol. 1997;37:631-7.

2. Mulliken JB, Glowacki J. Hemangiomas and vascular malformations in infants and children: a classification base don endothelial characteristics. Plast Reconstr Surg. 1982;69:412-22.

3. Bruckner AL, Frieden IJ. Hemangiomas of infancy. J Am Acad Dermatol. 2003;48:671-82.

4. Garzon MC, Enjolras O, Frieden IJ. Vascular tumors and vascular malformations: Evidence for an association. $\mathrm{J}$ Am Acad Dermatol. 2000;42:275-9.

5. Bruckner AL, Frieden IJ, Esterly NB. Infantile Hemangiomas and other vascular tumor. In: Harper $\mathrm{J}$, Orange A, Prose N, editors. Textbook of pediatric dermatology. Oxford: Blackwell Science; 2006. p. 1175-200.

6. Campos HGA. Anomalias Vasculares da Infância: avaliação de 10 anos de experiência do Hospital do Câncer (dissertação). São Paulo (SP): Fundação Antônio Prudente - Hospital A. C. Camargo Oncologia; 2000. p.136.

7. Wananukul S, Chatproedprai S. Ulcerated hemangiomas: clinical features and management. J Med Assoc Thai. 2002;85:1220-5.

8. Zarem HA, Edgerton MT. Induced resolution of cavernous hemangiomas following prednisolone therapy. Plast Reconstr Surg. 1967;39:76.

9. Frieden IJ, Haggstrom AN, Drolet BA, Mancini AJ, Friedlander SF, Boon L, et al. Infantile Hemangiomas: Current Knowledge, Futyre Directions. Proceedings of a Research Workshop on Infantile Hemangiomas. Pediatric Dermatol. 2005;22:383-406.

10. Delesalle F, Staumont D, Houmany MA, Brieviere GM, Piette F. Pulse methylprednisolone therapy for threatening periocular haemangiomas of infancy. Acta Derm Venereol. 2006; 86:429-32.

11. Garzon MC, Lucky AW, Hawrot A, Frieden IJ. Ultrapotent topical corticosteroid treatment of hemangiomas of infancy. J Am Acad Dermatol. 2005;52:281-6.

12. Ranchod TM, Frieden IJ, Fredrick DR. Corticosteroid treatment of periorbital haemangioma of infancy: a review of evidence. Br J Ophthalmol. 2005;89:1134-8.

13. Gontijo B, Silva CMR, Pereira LB. Hemangiomas na Infância. An Bras Dermatol. 2003;78:651-73.

14. Libby Edwards MD. Systemic dermatologic therapy the interferons. Dermatologic Clinics. 2001;19:139-46.

15. Zvulunov A, Metzker A. Hemangiomas and vascular malformations: unapproved treatments. Clin in Dermatol. 2002;20:660-7.

16. Mulliken JB, Rogers GF, Marler JJ. Circular excision of hemangioma and purse-string closure: The smallest possible scar. Plast Reconstr Surg. 2002;109:1544-54.

17. Gadelha AR, Costa IMC. Cirurgia Dermatológica em
Consultório. In: Souza JJ. Criocirurgia de lesões benignas e pré-malignas. São Paulo: Ed. Atheneu; 2003. p. 361-6.

18. Winter H, Drager E, Sterry W. Sclerotherapy for treatment of hemangiomas. Dermatol Surg. 2000;26:105-8.

19. Martinez MI, Sanchez-Carpintero I, North PE, Mihm MC Jr. Infantile hemangioma clinical resolution with 5\% Imiquimod cream. Arch Dermatol. 2002;138:881-4.

20. Welsh O, Olazarán Z, Gómez M, Salas J, Berman B. Treatment of infantile hemangiomas with short-term application of Imiquimod 5\% cream. J Am Acad Dermatol. 2004;51:639-42.

21. Doorne LV, Maeseneer M, Stricker C, Vanrensbergen R, Stricker M. Diagnosis and treatment of vascular lesions of the lip. Br J Oral Maxillofac Surg. 2002;40:497-503.

22. Burton BK, Schulz CJ, Angle B, Burd LI. An increased incidence of haemangiomas in infants born following chorionic villus sampling (CVS). Prenat Diagn. 1995; 15:209-14.

23. Garzon MC. Infantile hemangiomas. In: Bolognia $\mathrm{J}$, Jorizzo J, Rapini RP, eds. Textbook of dermatology. New York: Mosby vol II; 2003. p. 1599-1614.

24. Goldenberg DC, Cristofani LM, Almeida MTA, Odone Filho V, Ferreira MC. Tratamento dos hemangiomas cutâneos. Pediatr (SP). 2001;1:45-51.

25. Della Nina BI, Oliveira ZN, Machado MC, Macea JM. Apresentação, evolução e tratamento dos hemangiomas cutâneos - Experiência do Ambulatório de Dermatologia Infantil do Hospital das Clínicas da Faculdade de Medicina da Universidade de São Paulo. An Bras Dermatol. 2006;81:323-7.

26. Dutra AL. Avaliação do tratamento dos hemangiomas da Região Orbitária (dissertação). São Paulo (SP): Fundação Antônio Prudente - Hospital A. C. Camargo Oncologia; 2003. p.99.

27. Bennett ML, Fleisher AB Jr, Frieden IJ. Oral Corticosteroid use is Effective for Cutaneous Hemangiomas: An Evidence-Based Evaluation. Arch Dermatol. 2001;137:1208-13.

28. Chen MT, Yeong EK, Horng SY. Intralesional corticosteroid therapy in proliferating head and neck hemangiomas: A review of 155 cases. J Pediatr Surg. 2000;35:420-3.

29. Poetke M, Berlien HP. Laser treatment in hemangiomas and vascular malformations. Medical Laser Application. 2005;20:95-102.

30. Ho NT, Lansang P, Pope E. Topical imiquimod in the treatment of infantile hemangiomas: A retrospective study. J Am Acad Dermatol. 2007;56:63-8.

ENDEREÇO PARA CORRESPONDÊNCIA / MAILING ADDRESS:

Adriana Maria da Silva Serra

Endereço: SHIN QI 13 conj. 01 casa 10 Lago Norte

71.535010 Brasília DF

Tel./Fax: 6133262812 / 81368660 / 33262812

E-mail: amsserra@gmail.com

Como citar este artigo/How to cite this article: Serra AMS, Soares FMG, Cunha Jr AG, Costa IMC. Abordagem terapêutica dos hemangiomas cutâneos na infância. An Bras Dermatol. 2010;85(3):307-17. 